\title{
Flecainide Augments Muscle Sympathetic Nerve Activity in Humans
}

\author{
Yoshiki Nagata, MD
}

\begin{abstract}
The Cardiac Arrhythmia Suppression Trial has shown that treatment with flecainide is associated with an increased incidence of cardiac death in patients following myocardial infarction. It is believed that there is a complex mechanism involving an interaction between flecainide, sympathetic activation, and acute ischemia that is responsible for the increased risk of sudden death. The purpose of this study was to determine the effects of flecainide on muscle sympathetic nerve activity (MSNA) in humans. We measured MSNA using microneurography and cardiac output using the dye dilution method in 30 healthy individuals. Measurements were made at rest and after the oral administration of flecainide $(200 \mathrm{mg}, \mathrm{n}=12)$ or placebo $(\mathrm{n}=9)$, or intravenous administration of propranolol $(0.2 \mathrm{mg} / \mathrm{kg}, \mathrm{n}=9)$. Flecainide significantly increased heart rate and decreased the cardiac index (both $\mathrm{p}<0.01$ ). Flecainide increased the burst rate from $16.7 \pm 3.5$ to $23.3 \pm 4.1 \mathrm{bursts} / \mathrm{min}$ and the burst incidence from $26.6 \pm 5.1$ to $34.7 \pm 5.6$ bursts $/ 100$ heartbeats (both $\mathrm{p}<0.01$ ). For all of the hemodynamic parameters except heart rate, the effects of propranolol were similar to those of flecainide. Propranolol also increased the burst rate by $52 \pm 34 \%$ and the burst incidence by $106 \pm 39 \%$. These results suggest that flecainide suppresses myocardial contractility and produces reflex-mediated increases in sympathetic nerve firing in humans. (Circ J 2002; 66: 377-381)
\end{abstract}

Key Words: Flecainide acetate; Muscle sympathetic nerve activity; Negative inotropic action; Propranolol hydrochloride

$\mathbf{T}$ he Cardiac Arrhythmia Suppression Trial (CAST) was designed to test the hypothesis that suppression of ventricular ectopy after myocardial infarction decreases the incidence of arrhythmic death. However, an increased incidence in the number of deaths due to arrhythmia as well as deaths due to shock after recurrent myocardial infarction in patients treated with flecainide or encainide has been reported! Although the underlying mechanism for these adverse outcomes is unknown, subgroup analysis found that the total death and cardiac arrest rates were significantly higher in patients with non-Q-wave myocardial infarction, suggesting that ischemia plays an important role in the increased anti-arrhythmic drug risk?

Several subgroup analyses of the CAST data have also provided evidence supporting an additional contribution from sympathetic activity. These data demonstrated that the time of arrhythmic death in the group treated with flecainide or encainide displayed a bimodal variation, with significant peaks in the midmorning and late afternoon? Furthermore, CAST patients treated with both anti-arrhythmic drugs and $\beta$-blocker therapy had significantly better long-term survival than patients who received only anti-arrhythmic drugs4 Recently, Cragun et al have reported that $\beta$-adrenergic stimulation in the setting of membrane depolarization potentiated flecainide-induced conduction slowing, and have suggested that the synergy between catecholamines

(Received October 29, 2001; revised manuscript received January 7 , 2002; accepted January 17, 2002)

Department of Cancer Gene Regulation, Gastroenterology and Nephrology, Graduate School of Medical Science, Kanazawa University, Kanazawa, Japan

Mailing address: Yoshiki Nagata, MD, Department of Cancer Gene Regulation, Gastroenterology and Nephrology, Graduate School of Medical Science, Kanazawa University, 13-1 Takara-machi, Kanazawa 920-8641, Japan. E-mail: drnagata@ nsknet.or.jp and flecainide facilitates arrhythmogenesis in the presence of underlying ischemia5. It has also been shown in animal studies $^{6,7}$ and clinical studies ${ }^{8}$ that propranolol prevents the proarrhythmic effect of flecainide. These studies suggest an interaction between flecainide and the sympathetic nervous system in the development of arrhythmias.

The evaluation of the effects of anti-arrhythmic drugs on the sympathetic nervous system has received little attention. Certain anti-arrhythmic drugs have been shown to influence muscle sympathetic nerve activity (MSNA) directly or via reflex mechanisms 9,10 Although flecainide has been shown to produce a significant and marked reduction in heart rate variability,11,12 there have been no studis of the direct effects of flecainide on MSNA in humans.

In the present study, we hypothesized that flecainide suppresses myocardial contractility ${ }^{13,14}$ and might produce reflex-mediated increases in sympathetic nerve firing in humans. Therefore, we directly recorded efferent postganglionic sympathetic nerve activity in the peroneal nerve before and after oral administration of flecainide. Sympathetic nerve activity recorded after flecainide administration was then compared with propranolol administration producing comparable negative inotropic effects and placebo.

\section{Methods}

\section{Study Population}

The study included 30 healthy volunteers (20 men and 10 women; mean age $27 \pm 2$ years; range $19-54$ years). Subjects were screened by physical examination, biochemical and hematological analysis, and electrocardiogram. All subjects were of average physical fitness, and none were taking medication at the time. The experimental protocol and its purpose were explained in detail to each subject, and informed written consent was obtained. The study protocol 
Table 1 Baseline Clinical and Hemodynamic Characteristics

\begin{tabular}{lccc}
\hline \hline Variable & Control group & Flecainide group & Propranolol group \\
\hline No. of subjects & 9 & 12 & 9 \\
M/F & $6 / 3$ & $9 / 3$ & $5 / 4$ \\
Age (years) & $24 \pm 2$ & $31 \pm 4$ & $24 \pm 3$ \\
Heart rate (beats/min) & $65 \pm 4$ & $62 \pm 4$ & $65 \pm 3$ \\
Systolic arterial pressure $(\mathrm{mmHg})$ & $123 \pm 3$ & $136 \pm 4$ & $138 \pm 5$ \\
Diastolic arterial pressure $(\mathrm{mmHg})$ & $73 \pm 3$ & $77 \pm 2$ & $79 \pm 3$ \\
Cardiac index $\left(L \cdot \mathrm{min}^{-1} \cdot \mathrm{m}^{-2}\right)$ & $2.91 \pm 0.22$ & $3.14 \pm 0.27$ & $3.02 \pm 0.17$ \\
SVRI $($ units) & $2,565 \pm 183$ & $2,577 \pm 187$ & $2,679 \pm 204$ \\
\hline
\end{tabular}

Results are expressed as the mean \pm SEM. SVRI, systemic vascular resistance index.

was approved by the ethical panel of the Department of Cancer Gene Regulation, Gastroenterology and Nephrology, Graduate School of Medical Science, Kanazawa University.

\section{Measurements}

All experiments were carried out in a quiet room with subjects in a supine position. Arterial pressure was recorded directly from a polyethylene catheter inserted into the brachial artery and connected to a pressure transducer (Baxter UK1000; Baxter, Tokyo, Japan). Another polyethylene catheter was inserted into the antecubital vein for drug infusion. Heart rate was determined from a continuous recording of the electrocardiogram. Cardiac index was measured by the dye dilution method using a cuvette (TL-430S; Nihon Koden, Tokyo, Japan). Systemic vascular resistance index was calculated by dividing the mean arterial pressure (diastolic pressure plus one-third of the pulse pressure) by the cardiac index.

Multiunit postganglionic MSNA was recorded from a muscle nerve fascicle in the peroneal nerve at the level of the fibular head using tungsten microelectrodes (FHC Inc, Brunswick, USA) and microneurographic techniques! 15,16 The electrodes were connected to a preamplifier at a gain of 1,000 and an amplifier at a gain of 70. The signal was fed through a band-pass filter $(700-2,000 \mathrm{~Hz})$ and a resistancecapacitance integrating circuit with a time constant of $0.1 \mathrm{~s}$, producing a mean voltage neurogram. The signal was fed through a loudspeaker, displayed on an oscilloscope, and recorded by a paper chart recorder (Nihon Koden). The MSNA was identified based on its relationship to cardiac and respiratory activity, its tendency to increase during the Valsalva maneuver, and its lack of change during arousal stimuli and skin stroking. Sympathetic bursts were determined by inspection of the filtered and mean voltage neurogram. Nerve activity was expressed as both bursts/min (burst rate) and bursts/100 heartbeats (burst incidence).

\section{Study Protocol}

After placement of the intra-arterial catheter and microelectrodes for nerve activity recording, subjects were allowed to rest for $15 \mathrm{~min}$. Baseline data were recorded for $5 \mathrm{~min}$, and baseline values for arterial pressure, heart rate, and MSNA were averaged over the 5-min period. At least 2 measurements of cardiac output were obtained for each subject and an average value was used for comparison. All subjects performed a sustained handgrip exercise at $30 \%$ of maximal voluntary contraction for $3 \mathrm{~min}$.

After a 10-min recovery period, 12 subjects were given $200 \mathrm{mg}$ of flecainide orally. All measurements were repeated $60 \mathrm{~min}$ after the administration of flecainide. The same measurements were repeated $30 \mathrm{~min}$ after the intravenous administration of propranolol $(0.2 \mathrm{mg} / \mathrm{kg})$ in 9 subjects, and
$30 \mathrm{~min}$ and $60 \mathrm{~min}$ after the oral administration of placebo in 9 subjects. This dosing regimen has been shown to produce significant hemodynamic effects and significant increased plasma drug levels of flecainide ${ }^{14}$ and propranolol ${ }^{17,18}$

\section{Statistical Analysis}

Results are expressed as the mean \pm SEM. Student's ttest was used for paired comparisons. Repeated measures analysis of variance was used to compare within-group measurements. A value of $\mathrm{p}<0.05$ was considered statistically significant.

\section{Results}

\section{Subject Characteristics}

Table 1 summarizes the clinical characteristics of the 3 groups. The mean age of the flecainide group was slightly but insignificantly greater than that of the control group and the propranolol group. There was no significant difference in gender, resting heart rate, systolic and diastolic arterial pressure, cardiac index, or systemic vascular resistance index among the 3 groups.

\section{Hemodynamic Effects of Flecainide and Propranolol}

Table 2 summarizes the hemodynamic effects of flecainide and propranolol. Flecainide increased heart rate and diastolic arterial pressure (both $\mathrm{p}<0.01$ ) significantly, but did not affect systolic arterial pressure. After the oral administration of flecainide, the cardiac index decreased by $14 \pm 2 \%(\mathrm{p}<0.01)$, and was associated with a $17 \pm 6 \%$ increase in the systemic vascular resistance index. For all of the hemodynamic parameters except heart rate, the effects of propranolol were similar to those of flecainide. Propranolol decreased heart rate by $12 \pm 3 \%(\mathrm{p}<0.01)$. After the administration of placebo, there were no significant changes for any of the hemodynamic parameters.

\section{Effects of Flecainide and Propranolol on Muscle Sympathetic Nerve Activity}

Fig 1 shows a representative integrated neurogram, arterial pressure, and electrocardiogram. After the oral administration of flecainide, a marked increase in muscle sympathetic nerve bursts is noted. Flecainide significantly increased the burst rate by $98 \pm 56 \%(\mathrm{p}<0.01$; Table 3$)$ and the burst incidence by $79 \pm 46 \%(\mathrm{p}<0.01)$. Propranolol also increased the burst rate by $82 \pm 34 \%(\mathrm{p}<0.05$; Table 3$)$ and the burst incidence by $106 \pm 39 \%(\mathrm{p}<0.01)$. Placebo did not affect MSNA.

\section{Muscle Sympathetic Nerve Activity Responses to Handgrip Exercise}

During handgrip exercise (Fig 2), the increase in burst 
Table 2 Hemodynamic Effects of Flecainide, Propranolol and Placebo

\begin{tabular}{|c|c|c|c|c|c|c|c|}
\hline & \multicolumn{3}{|c|}{ Control group } & \multicolumn{2}{|c|}{ Flecainide group } & \multicolumn{2}{|c|}{ Propranolol group } \\
\hline & Baseline & $30 \mathrm{~min}$ & $60 \mathrm{~min}$ & Baseline & Flecainide & Baseline & Propranolol \\
\hline Heart rate (beats/min) & $65 \pm 4$ & $62 \pm 3$ & $63 \pm 3$ & $62 \pm 4$ & $67 \pm 4{ }^{\dagger}$ & $65 \pm 3$ & $57 \pm 2 \dagger$ \\
\hline Systolic arterial pressure $(\mathrm{mmHg})$ & $123 \pm 3$ & $122 \pm 2$ & $125 \pm 2$ & $136 \pm 4$ & $139 \pm 5$ & $138 \pm 5$ & $139 \pm 4$ \\
\hline Diastolic arterial pressure $(\mathrm{mmHg})$ & $73 \pm 3$ & $71 \pm 2$ & $75 \pm 3$ & $76 \pm 3$ & $81 \pm 2^{\dagger}$ & $79 \pm 3$ & $83 \pm 2 *$ \\
\hline Cardiac index $\left(L \cdot \mathrm{min}^{-1} \cdot \mathrm{m}^{-2}\right)$ & $2.91 \pm 0.22$ & $2.75 \pm 0.21$ & $2.97 \pm 0.23$ & $3.14 \pm 0.27$ & $2.69 \pm 0.21^{\dagger}$ & $3.02 \pm 0.17$ & $2.31 \pm 0.10$ \\
\hline SVRI (units) & $2,565 \pm 183$ & $2,690 \pm 223$ & $2,612 \pm 219$ & $2,577 \pm 187$ & $3,121 \pm 263^{\S}$ & $2,679 \pm 204$ & $3,591 \pm 206^{\S}$ \\
\hline
\end{tabular}

Results are expressed as the mean \pm SEM. SVRI, systemic vascular resistance index. ${ }^{*} p<0.05,{ }^{\dagger} p<0.01,{ }^{s} p<0.001$ compared with values at baseline.

Table 3 Effects of Flecainide and Propranolol on Muscle Sympathetic Nerve Activity and Electrocardiographic Parameters

\begin{tabular}{|c|c|c|c|c|c|c|c|}
\hline & \multicolumn{3}{|c|}{ Control group } & \multicolumn{2}{|c|}{ Flecainide group } & \multicolumn{2}{|c|}{ Propranolol group } \\
\hline & Baseline & $30 \mathrm{~min}$ & $60 \mathrm{~min}$ & Baseline & Flecainide & Baseline & Propranolol \\
\hline Burst rate (bursts/min) & $15.1 \pm 2.0$ & $13.9 \pm 2.0$ & $14.6 \pm 2.6$ & $16.7 \pm 3.5$ & $23.3 \pm 4.1^{\dagger}$ & $10.8 \pm 2.3$ & $17.1 \pm 2.7 *$ \\
\hline Burst incidence (bursts/100 heartbeats) & $23.8 \pm 3.3$ & $22.8 \pm 3.7$ & $23.9 \pm 4.9$ & $26.6 \pm 5.1$ & $34.7 \pm 5.6^{\dagger}$ & $17.5 \pm 4.1$ & $30.9 \pm 5.2^{\dagger}$ \\
\hline$P R$ interval $(s)$ & $0.15 \pm 0.01$ & $0.16 \pm 0.01$ & $0.16 \pm 0.01$ & $0.15 \pm 0.01$ & $0.16 \pm 0.01$ & $0.16 \pm 0.01$ & $0.16 \pm 0.01$ \\
\hline QRS duration (s) & $0.081 \pm 0.003$ & $0.081 \pm 0.003$ & $0.084 \pm 0.003$ & $0.090 \pm 0.065$ & $0.103 \pm 0.005 *$ & $0.090 \pm 0.005$ & $0.090 \pm 0.005$ \\
\hline$Q T$ interval $(s)$ & $0.39 \pm 0.01$ & $0.39 \pm 0.01$ & $0.40 \pm 0.01$ & $0.39 \pm 0.01$ & $0.39 \pm 0.01$ & $0.41 \pm 0.01$ & $0.39 \pm 0.01^{\dagger}$ \\
\hline
\end{tabular}

Results are expressed as the mean \pm SEM. ${ }^{*} p<0.05,{ }^{\dagger} p<0.01$ compared with values at baseline.

Baseline

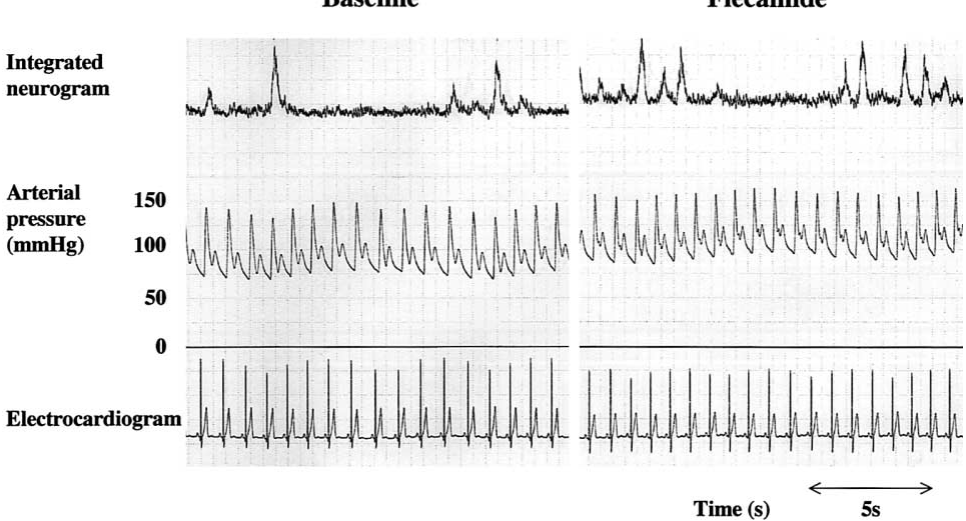

Fig 1. Typical example of experimental records showing the effects of oral administration of flecainide on muscle sympathetic nerve activity, arterial pressure, and electrocardiogram (ECG). incidence under basal conditions was $7.2 \pm 2.0$ bursts/100 heartbeats, which was similar to that after the administration of flecainide $(9.7 \pm 2.5$ bursts/100 heartbeats). In the propranolol and placebo group, handgrip exercise led to similar increases in burst incidence under basal conditions and after drug administration.

\section{Electrocardiogram}

Flecainide significantly increased the QRS duration ( $p<0.05$; Table 3), but did not affect the PR or QT intervals. After propranolol infusion, the QT interval decreased ( $\mathrm{p}<0.01$; Table 3$)$, but the QRS duration and PR interval did not change. There was no significant change in the electrocardiographic parameter after the administration of placebo.

\section{Discussion}

The present study is the first to demonstrate that flecainide enhances sympathetic outflow in humans from direct measurements of sympathetic nerve activity in the peroneal nerve.

\section{Anti-Arrhythmic Drugs and Sympathetic Nerve Activity}

The sympathetic nervous system is an important regulator of cardiovascular function. Activation of the sympa-

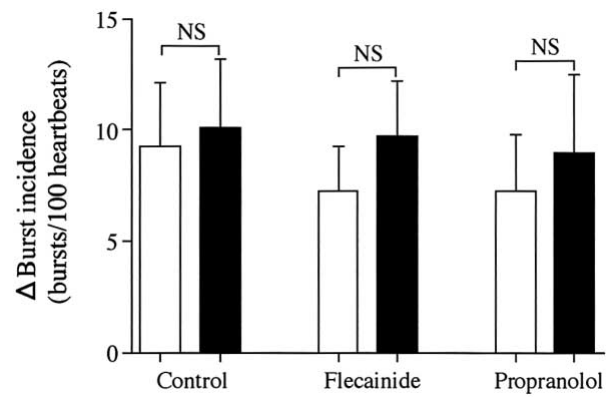

Fig 2. Increases in burst incidence during handgrip exercise at ( $\square$ ) baseline, and (匹) after administration of placebo, flecainide, and propranolol. Values are expressed as the mean \pm SEM. NS, not significant.

thetic nervous system plays an important role in the pathophysiology and prognosis of cardiovascular disorders such as heart failure, coronary heart disease, hypertension, and arrhythmias. ${ }^{19-23}$ Clinical trials of short-acting calcium channel antagonists have yielded disappointing results, particularly in patients with acute myocardial infarction and unstable angina ${ }^{24} \mathrm{~A}$ potential mediator of these untoward effects is activation of the sympathetic nervous system. 25 Therefore, an analysis of the interaction between 
cardiovascular drugs and the sympathetic nervous system is important.

Certain anti-arrhythmic drugs have been shown to influence MSNA, either directly or via a reflex mechanism. Rea et al have reported that the intravenous administration of procainamide decreases postganglionic sympathetic nerve activity in humans directly and that the vasodilator effects of the drug may be because of withdrawal of the sympathetic vasoconstrictor tone? Mariano et al have demonstrated that quinidine, unlike procainamide, causes vasodilation directly and is associated with baroreflex-mediated increases in sympathetic nerve activity 10 Furthermore, Ellenbogen and colleagues have demonstrated that therapeutic doses of lidocaine do not have a direct effect on MSNA during sustained ventricular tachycardia or during programmed electrical stimulation with single ventricular extrastimuli 26 The results of the present study demonstrate, for the first time, that flecainide increases sympathetic outflow in humans. In addition, flecainide increases the heart rate and systemic vascular resistance index, suggesting cardiac and peripheral sympathetic augmentation.

The autonomic effects of flecainide have not been investigated in great detail. It has been shown that flecainide decreases heart rate variability!11,12 The potential importance of the interaction between flecainide and activation of the sympathetic nervous system has also been suggested by the observation that the proarrhythmic effect of flecainide can be prevented by $\beta$-blocking agents ${ }^{6-8}$

\section{Potential Mechanisms}

Although the exact mechanisms by which flecainide enhances sympathetic nerve activity remain unclear, the following 2 mechanisms should be considered: (1) suppression of afferent vagal nerve activity through negative inotropic actions, and (2) direct action on the central nervous system.

Cardiopulmonary mechanoreceptors with C-fiber vagal afferents have been shown to inhibit sympathetic vasomotor outflow tonically in animal studies 27,28 Studies in openchest, anesthetized cats have indicated that the 2 main mechanical determinants of ventricular C-fiber activity are left ventricular end-diastolic pressure and contractility? 7 Decreases in ventricular contractility by acute $\beta$-adrenergic blockade attenuate the increase in C-fiber activity caused by increases in end-diastolic pressure ${ }^{27,28}$ In the present study, we hypothesized that flecainide decreases myocardial contractility ${ }^{13,14}$ and might produce reflex-mediated increases in sympathetic nerve firing. Therefore, we compared sympathetic nerve activity after flecainide administration with that after the intravenous infusion of propranolol at a dose that produced comparable negative inotropic effects. In the present study, flecainide decreased the cardiac index to an extent similar to propranolol. The degree of activation of the muscle sympathetic nervous system between flecainide and propranolol was similar. These results suggest that flecainide causes increased reflex in sympathetic nerve activity through an inhibition of cardiopulmonary baroreceptor discharge. Jacobsen et al have found no increase in muscle sympathetic activity in humans following the intravenous injection of propranolol at a dose of $0.15 \mathrm{mg} / \mathrm{kg}^{29}$ However, recent laboratory studies of the spectral analysis of heart rate, arterial pressure, and muscle sympathetic activity in normal humans found that propranolol $(0.2 \mathrm{mg} / \mathrm{kg}$ iv) increases burst incidence from $19.0 \pm 2.7$ to $28.1 \pm 2.3$ bursts/100 heartbeats 30 The discrepan- cies between studies of the effect of propranolol are likely to be related to differences in propranolol dosage.

The direct stimulation of the sympathetic nervous system through a central mechanism by flecainide is unlikely. This is based indirectly on the finding that flecainide had no effect on the response of MSNA to handgrip exercise. Furthermore, there are no previously published experimental data supporting such an interaction? ${ }^{1}$

\section{Study's Limitations}

The present study has several limitations. First, we measured MSNA but did not evaluate cardiac sympathetic activity. However, there is evidence indicating that in healthy normotensive individuals, there is a close relationship between resting MSNA in the peroneal nerve and cardiac norepinephrine spillover ${ }^{32}$ In the present study we found that flecainide increased heart rate; therefore, flecainide may enhance cardiac sympathetic drive as well as muscle.

Second, the study does not determine whether these sympathetic effects persist during chronic oral flecainide treatment in patients with cardiovascular diseases. Further studies are necessary to assess this aspect of the effects of flecainide.

\section{Clinical Implications}

Despite the fact that the results of this short-term study were obtained in healthy volunteers, the treatment strategies used may be relevant to patients with cardiovascular disease in whom sympathetic activation is the major determinant of prognosis. Previous studies have demonstrated circadian rhythms in the onset of sudden cardiac death, myocardial infarction, and transient myocardial ischemia, and have suggested that increased sympathetic activity associated with awakening and rising may be involved20,33-37 In the CAST, the time of onset of arrhythmic death in the active treatment group displayed a bimodal variation, with significant peaks in the midmorning and late afternoon/early evening? It is likely that flecainide-induced sympathetic activation produced the detrimental effects in this group. Based on the results of the present study, the selection of anti-arrhythmic drugs for the treatment of arrhythmias in patients with myocardial infarction should also take into consideration their effects on the sympathetic nervous system.

\section{Conclusions}

The results suggest that flecainide suppresses myocardial contractility and produces reflex-mediated increases in sympathetic nerve firing in humans. This sympathetic excitation is likely to be a result of diminished cardiac output.

\section{Acknowledgments}

I thank Professor K Kobayashi (Department of Cancer Gene Regulation, Gastroenterology and Nephrology, Graduate School of Medical Science, Kanazawa University) and Prof. S Takata (Department of Health Science, Graduate School of Medical Science, Kanazawa University), both of whom gave me a lot of encouragement and helped in the design of the study. I also thank the Cardiology group at the Department of Cancer Gene Regulation, which assisted me with the technical methods.

\section{References}

1. Echt DS, Liebson PR, Mitchell LB, Peters RW, Obias-Manno D, Barker AH, et al and Cardiac Arrhythmia Suppression Trial investi- 
gators. Mortality and morbidity in patients receiving encainide, flecainide, or placebo. The Cardiac Arrhythmia Suppression Trial. $N$ Engl J Med 1991; 324: 781-788.

2. Anderson JL, Platia EV, Hallstrom A, Henthorn RW, Buckingham TA, Carlson MD, et al and Cardiac Arrhythmia Suppression Trial investigators. Interaction of baseline characteristics with the hazard of encainide, flecainide, and moricizine therapy in patients with myocardial infarction: A possible explanation for increased mortality in the Cardiac Arrhythmia Suppression Trial (CAST). Circulation 1994; 90: 2843-2852

3. Peters RW, Mitchell LB, Brooks MM, Echt DS, Barker AH, Capone $\mathrm{R}$, et al and Cardiac Arrhythmia Suppression Trial investigators. Circadian pattern of arrhythmic death in patients receiving encainide, flecainide or moricizine in the cardiac arrhythmia suppression trial (CAST). J Am Coll Cardiol 1994; 23: 283-289.

4. Kennedy HL, Brooks MM, Barker AH, Bergstrand R, Huther ML, Beanlands DS, et al and Cardiac Arrhythmia Suppression Trial investigators. Beta-blocker therapy in the cardiac arrhythmia suppression trial. Am J Cardiol 1994; 74: 674-680.

5. Cragun KT, Johnson SB, Packer DL. $\beta$-Adrenergic augmentation of flecainide-induced conduction slowing in canine Purkinje fibers. Circulation 1997; 96: 2701-2708.

6. Dhein S, Müller A, Gerwin R, Klaus W. Comparative study on the proarrhythmic effects of some antiarrhythmic agents. Circulation 1993; 87: 617-630

7. Stramba-Badiale M, Lazzarotti M, Facchini M, Schwartz PJ. Malignant arrhythmias and acute myocardial ischemia: Interaction between flecainide and the autonomic nervous system. Am Heart J 1994; 128: 973-982.

8. Myerburg RJ, Kessler KM, Cox MM, Huikuri H, Terracall E, Interian A, et al. Reversal of proarrhythmic effects of flecainide acetate and encainide hydrochloride by propranolol. Circulation 1989; 80: 15711579.

9. Rea RF, Hamdan M, Schomer SJ, Geraets DR. Inhibitory effects of procainamide on sympathetic nerve activity in humans. Circ Res 1991; 69: $501-508$.

10. Mariano DJ, Schomer SJ, Rea RF. Effects of quinidine on vascular resistance and sympathetic nerve activity in humans. $J$ Am Coll Cardiol 1992; 20: 1411-1416.

11. Zuanetti G, Latini R, Neilson JMM, Schwartz PJ, Ewing DJ and Antiarrhythmic Drug Evaluation Group (ADEG). Heart rate variability in patients with ventricular arrhythmias: Effect of antiarrhythmic drugs. J Am Coll Cardiol 1991; 17: 604-612.

12. Lombardi F, Torzillo D, Sandrone G, Vecchia LD, Cappiello E. Autonomic effects of antiarrhythmic drugs and their importance. Eur Heart J 1992; 13(Suppl F): 38-43.

13. Josephson MA, Kaul S, Hopkins J, Kvam D, Singh BN. Hemodynamic effects of intravenous flecainide relative to the level of ventricular function in patients with coronary artery disease. $\mathrm{Am}$ Heart J 1985; 109: 41-45.

14. Nakamura T, Nezuo S, Hasegawa K, Tadaoka S, Nakao M, Kakumae $\mathrm{S}$, et al. Hemodynamic effects of flecainide in relation to its serum concentration. Kokyu to Jyunkan 1988; 36: 885-891.

15. Hagbarth KE, Vallbo ÅB. Pulse and respiratory grouping of sympathetic impulses in human muscle nerves. Acta Physiol Scand 1968; 74: $96-108$

16. Delius W, Hagbarth KE, Hongell A, Wallin BG. General characteristics of sympathetic activity in human muscle nerves. Acta Physiol Scand 1972; 84: 65-81.

17. Ogawa J, Takata S, Aoki K, Oukuwa N, Iwase N, Ikeda T, et al. Acute changes in the properties of baroreflexes in man after $\beta$-blockade. Clin Exp Pharmacol Physiol Suppl 1989; 15: 125-128.

18. McDevitt DG, Shand DG. Plasma concentrations and the time- course of beta blockade due to propranolol. Clin Pharmacol Ther 1975; 18: 708-713.

19. Packer M, Lee WH, Kessler PD, Gottlieb SS, Bernstein JL, Kukin ML. Role of neurohormonal mechanisms in determining survival in patients with severe chronic heart failure. Circulation 1987; 75 (Suppl IV): IV-80-IV-92.

20. Muller JE, Stone PH, Turi ZG, Rutherford JD, Czeisler CA, Parker $\mathrm{C}$, et al and the Multicenter Investigation of Limitation of Infarct Size (MILIS) Study Group. Circadian variation in the frequency of onset of acute myocardial infarction. N Engl J Med 1985; 313: 1315-1322.

21. Julius S. Sympathetic hyperactivity and coronary risk in hypertension. Hypertension 1993; 21: 886-893.

22. Zipes DP, Wellens HJJ. Sudden cardiac death. Circulation 1998; 98: $2334-2351$.

23. Watanabe T, Kim S, Akishita M, Kario K, Sekiguchi H, Fujikawa H, et al. Circadian variation of autonomic nervous activity in patients with multivessel coronary spasm. Jpn Circ J 2001; 65: 593-598.

24. Held PH, Yusuf S, Furberg CD. Calcium channel blockers in acutemyocardial infarction and unstable angina: An overview. $\mathrm{Br}$ Med $\mathrm{J}$ 1989; 299: 1187-1192.

25. Wenzel RR, Allegranza G, Binggeli C, Shaw S, Weidmann P, Lüscher TF, et al. Differential activation of cardiac and peripheral sympathetic nervous system by nifedipine: Role of pharmacokinetics. J Am Coll Cardiol 1997; 29: 1607-1614.

26. Ellenbogen KA, Smith ML, Beightol LA, Eckberg DL. Influence of lidocaine on human muscle sympathetic nerve activity during programmed electrical stimulation and ventricular tachycardia. Am Heart J 1992; 124: 891 -897.

27. Thorén PN. Characteristics of left ventricular receptors with nonmedullated vagal afferents in cats. Circ Res 1977; 40: 415-421.

28. Thames MD. Effect of d- and 1-propranolol on discharge of cardiac vagal C fibers. Am J Physiol 1980; 238: H465-H470.

29. Jacobsen TN, Converse Jr RL, Victor RG. Contrasting effects of propranolol on sympathetic nerve activity and vascular resistance during orthostatic stress. Circulation 1992; 85: 1072-1076.

30. Nakata A, Takata S, Yuasa T, Shimakura A, Maruyama M, Nagai H, et al. Spectral analysis of heart rate, arterial pressure, and muscle sympathetic nerve activity in normal humans. Am J Physiol 1998; 274: $\mathrm{H} 1211-\mathrm{H} 1217$

31. Cowan JC, Williams V. Characterization of a new oral antiarrhythmic drug, flecainide. Eur J Pharmocol 1981; 73: 333 -342.

32. Wallin BG, Esler M, Dorward P, Eisenhofer G, Ferrier C, Westerman $\mathrm{R}$, et al. Simultaneous measurements of cardiac noradrenaline spillover and sympathetic outflow to skeletal muscle in humans. J Physiol (Lond) 1992; 453: 45-58.

33. Muller JE, Ludmer PL, Willich SN, Tofler GH, Aylmer G, Klangos $\mathrm{I}$, et al. Circadian variation in the frequency of sudden cardiac death. Circulation 1987; 75: 131-138.

34. Willich SN, Levy D, Rocco MB, Tofler GH, Stone PH, Muller JE. Circadian variation in the incidence of sudden cardiac death in the Framingham Heart Study population. Am J Cardiol 1987; 60: 801 806.

35. Kranz DS, Kop WJ, Gabbay FH, Rozanski A, Branard M, Klein J, et al. Circadian variation of ambulatory myocardial ischemia: Triggering by daily activities and evidence for an endogenous circadian component. Circulation 1996; 93: 1364-1371.

36. Kong TQ, Goldberger JJ, Parker M, Wang T, Kadish AH. Circadian variation in human ventricular refractoriness. Circulation 1995; 92: $1507-1516$.

37. Itoh H, Shimizu M, Ino H, Okeie K, Yamaguchi M, Fujino N, et al and Hokuriku Brugada Study Group. Arrhythmias in patients with Brugada-type electrocardiographic findings. Jpn Circ J 2001; 65: $483-486$. 